\title{
Sur la solubilité en nombres entiers des équations du second degré à deux indéterminées
}

par

TRYGVE NAGELC (Uppsala)

\section{\$1. Introduction. Résultats antérieurs}

1. Pour certains ensembles d'équations diophantiennes à deux incléterminées il existe des résultats du type suivant: Parmi les équations appartenant an même ensemble il y a an plus une seule équation qui est résoluble en nombres entiers rationnels. Quelquefois il y a exactement deux équations résolubles.

$\Pi$ y a de tels résultats sur des ensembles d'équations quadratiques; voir [5] $\left.{ }^{1}\right)$ et [6]. Pour des résultats analogues sur les équations cubiques, biquadratiques et même de degré $>4$ voir [7], [3], [4] et [1]. Dans [8], \$ 4, j'ai donné un aperȩu de tous ces résultats.

2. Dans les travaux [5] et [6] j'ai établi le résultat suivant:

THÉoRtime 1. Soit donné le nombre naturel $D \geqslant 2$ qui n'est divisible par ancun carré $>1$. De plus, soient $A$ et $B$ des nombres naturels variables tels que $A B=D$ et $1 \leqslant A<B$. Pour toutes les valeurs permises de $A$. et $B$ nous considerons l'ensemble des équations

$$
A x^{2}-B y^{2}=E \text {, }
$$

ois $D=+1$ et +2 pour $D$ impair et $E= \pm 1$ pour $D$ pair.

Alors, abstraction faite de l'equation

$$
x^{2}-D y^{2}=1
$$

it y a dans cet ensemble exactement une et une seule équation qui est résoluble on nombres naturels $x$ et $y$.

Cette équation résoluble, distincte de l'équation (2), sera appelée l'équation singutière de l'ensemble. Dans ce qui suivra nous la désignerons par

$$
\text { (3) } \quad A^{*} x^{2}-B^{*} y^{2}=E^{*} \text {, }
$$

où $A^{*} B^{*}=D, 1 \leqslant A^{*}<B^{*}$ et $B^{*}= \pm 1$ ou \pm 2 .

(1) Les munéros figurant entre crochots renvoient à la bibliographie planée à la tin do ce mémoire. 
Lorsque $D$ est impair le nombre $E^{*}$ peut prendre toutes les quatre valeurs possibles, ainsi qu'on le voit des exemples suivants;

$$
\begin{array}{ll}
D=3, & x^{2}-3 y^{2}=-2 ; \\
D=5, & x^{2}-5 y^{2}=-1 ; \\
D=7, & x^{2}-7 y^{2}=+2 ; \\
D=33, & 3 x^{2}-11 y^{2}=+1 .
\end{array}
$$

Pour $D$ pair, on a les exemples suivants:

$$
\begin{array}{ll}
D=6, & 2 x^{2}-3 y^{2}=-1 ; \\
D=14, & 2 x^{2}-7 y^{2}=+1 .
\end{array}
$$

Nous désignons l'ensemble défini dans le Théorème 1 par le srmbole $[D, A, B ; E]$. Si $D$ est le produit de $r$ nombres premiers, le nombre d'équations dans cet ensemble est $=2^{r+1}$ ou $=2^{r}$ suivant que $D$ est impair ou pair.

Un résultat analogue est donné par le

THÉoRèce 2. Soit donné le nombre naturel $D=5(\bmod 8)$ qui n'est divisible par aucun carré $>1$. De plus, soient $A$ et $B$ des nombres naturels variables tels que $A B=D$ ot $1 \leqslant A<B$. Supposons que l'équation

$$
x^{2}-D y^{2}=4
$$

soit résoluble en nombres naturels impairs $x$ et $y$. Pour toutes les valeurs permises de $A$ et $B$ nous considérons l'ensemble des équations

$$
A x^{2}-B y^{2}= \pm 4
$$

Alors, abstraction faite de l'équation (4), il y a dans l'ensemble (5) exantement une et une seule équation qui est résoluble en nombres naturels impains ot of $\%$

Si $D$ est le produit de $r$ nombres premiers, le nombjo d'équations dans l'ensemble de ce théorème est $=2^{r}$.

On peut se demander s'il est possible de généraliseri lo Théorine 1. et établir un résultat analogue pour un ensemble d'équations

$$
A x^{2}-B y^{2}=E N
$$

où $A, B, D$ et $E$ ont les mêmes significations que dans le Théorimo L, et où $N$ est un nombre naturel impair $>1$.

Nous désignons l'ensemble ainsi défini par le symbole $[D, A, B ; E N]$.

Dans le paragraphe suivant nous allons traiter le cas où $N$ est thin nombre premier impair, qui ne divise pas $D$.

\section{§ 2. Les ensembles $[D, A, B ; E p]$}

3. Soient $D, A, B$ et $E$ des nombres définis comme dans le Théorème 1 , et soit $p$ un number premier fixe $\geqslant 3$ qui ne divise pas $D$. Cela étant, nous considérons l'ensemble $[D, A, B ; E p]$ constitué de toutes les équations permises de la forme

$$
A x^{2}-B y^{2}=E p \text {. }
$$

Parmi ces équations nous distinguons deux catégories: La première catégorie aveo $B= \pm 1$; la seconde catégorie avec $B= \pm 2$.

Supposons maintenant qu'il existe deux équations résolubles appartenant à la même catégorie, savoir

$$
\begin{gathered}
A x^{2}-B y^{2}=E p \\
A_{1} x_{1}^{2}-B_{1} y_{1}^{2}=B_{1} p .
\end{gathered}
$$

Alors on a $A B=A_{1} B_{1}=D, 1 \leqslant A<B, 1 \leqslant A_{1}<B_{1}$ et $|E|=\left|E_{1}\right|$. Les nombres $A x / y$ et $A_{1} x_{1} / y_{1}$ sont des solutions de la congruence

$$
z^{2} \equiv D(\bmod p) \text {. }
$$

Les signes de ces nombres peurent évidemment être choisis de façon qu'on ait

$$
\text { (8) } \quad A_{1} x_{1} y \equiv A x y_{1}(\bmod p) \text {. }
$$

On doit observer que $p$ ne divise pas le produit $x y x_{1} y_{1}$.

Considérons maintenant le produit

$$
\left[(A x)^{2}-D y^{2}\right]\left[\left(A_{1} x_{1}\right)^{2}-D y_{1}^{2}\right]=E E_{1} A A_{1} p^{2} .
$$

II peut s'écrire

$$
\left(A A_{1} x x_{1}-D y y_{1}\right)^{2}-D\left(A_{1} y x_{1}-A x y_{1}\right)^{2}=E E_{1} \cdot A \cdot A_{1} p^{2}
$$

Supposons ensuite que

$$
\left(A, A_{1}\right)=A_{2}, \quad A=A_{2} A_{3}, A_{1}=A_{2} A_{4},
$$

où $A_{2}, A_{3}$ et $A_{4}$ sont des nombres naturels, premiers entre eux deux à deux. Alors on obtient de (9) la relation

où les quantitiés

$$
A_{3} A_{4} U^{2}-\frac{D}{A_{3} A_{4}} V^{2}= \pm 1=\frac{\left|E E_{1}\right|}{E E_{1}}
$$

et

$$
\begin{gathered}
U=\frac{1}{E p}\left[A_{2} x x_{1}-\frac{D}{A A_{4}} y y_{1}\right] \\
V=\frac{1}{E p}\left[A_{3} x y_{1}-A_{4} x_{1} y\right]
\end{gathered}
$$


sont des nombres entiers en vertu de la congruence (8) et en vertu, du fait que pour $|\mathbb{E}|=\left|E_{1}\right|=2$ tous les nombres $D, A, A_{1}, A_{2}, A_{3}, A_{4}$, $x, y, x_{1}$ et $y_{1}$ sont impairs.

Pour l'équation (10) il existe deux possibilités: Ou elle coïncide arec l'équation $x^{2}-D y^{2}=1$, ou elle coincide avoc l'équation singulière (3) de l'ensemble $[D, A, B ; E]$. Vu que dans $(10)$ le terme à droite est \pm 1 , le dernier cas se présente seulement lorsque $E^{*}= \pm 1$; la possibilité $E^{*}= \pm 2$ est donc exclue dans le présent cas.

Il y aura dono deux possibilités à examiner. Nous allons d'abord montrer que le cas où l'équation (10) coïneide arec l'équation $x^{2}-D y^{2}=1$ ne pent exister.

4. Premier cas. L'équation (10) cöncide avec l'équation $D^{2} \ldots D V^{2}=x$. Si on prend le signe inférieur dans (10) il faut que $D=A_{8} A_{4}$. Done

$$
B B_{1}=\frac{D^{2}}{A_{2}^{2} A_{3} A_{4}}=\frac{D}{A_{2}^{2}},
$$

ce qui entraîne $A_{2}=1$ et par suite $B B_{1}=D$, d'où $B=A_{1}$ et $B_{1}=A$. Or, cela est impossible vu que $1 \leqslant A<B$ et $1 \leqslant A_{1}<B_{1}$.

Prenons ensuite le signe supérieur dans (10). Alors il faut que. $A_{3} A_{4}=1$. Donc $A_{3}=A_{4}=1$ et $A=A_{1}$ et par suite $B=B_{1}$. Cela entraîne que $E_{1}=-E$.

Donc, l'équation (7) aura la forme

$$
A x_{1}^{2}-B y_{1}^{2}=-E p \text {. }
$$

Cependant, celà entraịne que le terme à droite dans (10) soit égal à -1 . Or, nous venons de montrer qu'on ne pent pas avoir lo signe inférieur dans (10).

Second cas. L'équation (10) cołncide avec l'équation singulière (3) de l'ensemble $[D, A, B ; E]$, savoir

$$
A^{*} U^{2}-B^{*} V^{2}=E^{*} .
$$

Ici on a $A^{*} B^{*}=D$. En supprimant la condition $1 \leqslant A^{*}<B^{*}$ nous pouvons supposer que $B^{*}=+1$. Le cas $A^{*}=1, B^{*}=D$, quo nous venons de traiter, peut être exclu. Sans rien perdro de la généralité nous pouvons supposer que $A \geqslant A_{1}$

Si on prend le signe supérieur dans (10) il faut que

$$
A^{*}=A_{3} A_{4} \quad \text { et } \quad B^{*}=\frac{D}{A_{8} A_{4}}=\frac{A B}{A_{8} A_{4}} .
$$

Il en résulte, vi que $\left(A_{2}, A_{4}\right)=1$,

$$
\left(A, A^{*}\right)=\left(A_{2} A_{3}, A_{3} A_{4}\right)=A_{3}
$$

et de plus

$$
\frac{B^{*}}{\left(B, B^{*}\right)}=A_{2} \frac{B}{A_{4}\left(B, B^{*}\right)} .
$$

On en conclut que

$$
B=A_{4}\left(B, B^{*}\right) \quad \text { et } \quad B_{1}=\frac{D}{A_{1}}=\frac{A B}{A_{2} A_{4}}=A_{3}\left(B, B^{*}\right) .
$$

Pair conséquent on aura

$$
\begin{aligned}
B_{1} & =\left(A, A^{*}\right)\left(B, B^{*}\right), \\
A_{1} & =\frac{D}{\left(A, A^{*}\right)\left(B, B^{*}\right)}
\end{aligned}
$$

Il faut contrôler qu'on a $A_{1}<B_{1}$. En effet, il résulte en vertu de la condition $A \geqslant A_{1}$ que $A_{3} \geqslant A_{4}$. Des formules (14) et (15) on obtient

$$
\frac{A_{1}}{B_{1}}=\frac{D}{\left(A, A^{*}\right)^{2}\left(B, B^{*}\right)^{2}}
$$

d'où, vu que

$$
\begin{gathered}
\left(A, A^{*}\right)=A_{3} \quad \text { et } \quad\left(B, B^{*}\right)=\frac{B}{A_{4}}, \\
\frac{A_{1}}{B_{1}}=\frac{A}{B}\left(\frac{A_{4}}{A_{3}}\right)^{2}<1 .
\end{gathered}
$$

Si on prend le signe inférieur dans (10) on aura au lieu des formules (14) et (15) les relations

$$
\begin{aligned}
& B_{1}=\left(A, B^{*}\right)\left(B, A^{*}\right), \\
& A_{1}=\frac{D}{\left(A, B^{*}\right)\left(B, A^{*}\right)} .
\end{aligned}
$$

Donc, $A_{1}$ et $B_{1}$ sont univoquement déterminés lorsque $A$ et $B$ sont données, par les formules (14) et (15) si $E=E_{1}$ et par les formules (16) et (17) sị $E=-E_{1} \cdot A^{*}$ et $B^{*}$ sont univoquement cléterminés lorsque $D$ est donné.

II résulte du raisonnement précédent qu'une troisième équation résoluble de la même catégorie ne peut pas exister.

S'il y a une équation résoluble dans l'ensemble, il y en a une seconde équation résoluble dans la même catégorie lorsque l'équation (13) est résoluble. En effet, lorsque les nombres $A, B, E, x, y, A^{*}, B^{*}, U$ et $\nabla$ sont connus et si $E=E_{1}$, on peut d'abord déterminer $A_{1}$ et $B_{1}$ à l'aide 
de formules (14) et (15) et puis $x_{1}$ et $y_{1}$ à l'aide des formules (11) et (12), qui premnent alors la forme

$$
\begin{aligned}
& E U p=\left(A, A^{*}\right) x x_{1}-\left(B, B^{*}\right) y y_{1}, \\
& E V p=\frac{A}{\left(A, A^{*}\right)} x y_{1}-\frac{B}{\left(B, B^{*}\right)} x_{1} y .
\end{aligned}
$$

Il en résulte

$$
\begin{aligned}
& x_{1}=\frac{A}{\left(A, A^{*}\right)} U x+\left(B, B^{*}\right) V y \\
& y_{1}=\frac{B}{\left(B, B^{*}\right)} U y+\left(A, \mathcal{A}^{*}\right) V x
\end{aligned}
$$

ce qui montre que $x_{1}$ et $y_{1}$ sont des nombres entiers.

Dans le cas de $E_{1}=-E$ il faut appliquer les formules (16) et (17) pour déterminer $A_{1}$ et $B_{1}$. Pour obtenir les valeurs de $x_{1}$ et $y_{1}$ dans ce cas on aura seulement d'échanger $A$ et $B$, ainsi que $U$ et $V$, dans les formules (18) et $\left(18^{\prime}\right)$.

Il est évident que la possibilité $A=A_{1}, B=B_{1}$ ne peut se présenter que pour $B_{1}=-E$.

5. Supposons maintenant que les deux équations suivantes de catégories différentes soient résolubles à la fois:

$$
\begin{gathered}
A x^{2}-B y^{2}=E p= \pm 2 p \\
A_{1} x_{1}^{2}-B_{1} y_{1}^{2}=E_{1} p= \pm p
\end{gathered}
$$

Alors tous les nombres $D, A, B, A_{1}$ et $B_{1}$ sont impairs. La congruence (8) pour les nombres $x, y, x_{1}$ et $y_{1}$ est toujours satisfaite.

En appliquant la même méthode que dans le numéro 3 sur le produit

$$
\left[(A x)^{2}-D y^{2}\right]\left[\left(A_{1} x_{1}\right)^{2}-D y_{1}^{2}\right]
$$

nous aurons une équation analogue à (10), de la forme

$$
A^{*} U^{2}-B^{*} V^{2}=E E_{1}= \pm 2,
$$

où $A^{*} B^{*}=D$, et où $V$ et $V$ sont des nombres entiers. Oette équation. est nécessairement l'équation singulière dans l'ensemble $[D, A, B ; D]$.

Nous venons de voị tout à l'heure que poư l'existence de deux équations de la même catégorie une condition nécessaire est que l'équation singulière de l'ensemble $[D, A, B ; E]$ soit de la form $\Theta$

$$
A^{*} U^{2}-B^{*} V^{2}= \pm 1 \text {. }
$$

On en conclut: S'il existe deux équations résolubles do catégories différentes, ces équations sont les seules résolubles dans l'ensemble $[D, A, B ; E p]$.

Par conséquent, nous avons établi le

THÉoRłime 3. Dans l'ensemble $[D, A, B ; E p]$ il y a exactement deux équations résolubles, s'il y en a une. Il y a les quatre possibilités suivantes: $1^{\circ} I l$ n'y a aucune équation résoluble; $2^{\circ}$ Deux équations de la première catégorie sont résolubles; $3^{\circ}$ Deux équations de la seconde catégorie sont résolubles; $4^{\circ}$ Une seule équation de chacune des deux catégories est résoluble.

On peut d'ailleurs établị̂ ce résultat à l'aide de la théorie des formes binaires quadratiques. Cependant la présente méthode est evidemment plus simple.

Pour déterminer les équations résolubles dans un ensemble donné on pent p.ex. appliquer la méthode que j'ai devéloppée dans mon livre [9], Chapter VI, no. 58.

Notons le résultat suivant:

Soit donné le nombre naturel $D$ qui est le produit de nombres premiers différents. Alors il existe une infinité de nombres premiers $p$, tels que l'ensemble $[D, A, B ; E p]$ contienne deux équations résolubles.

On le vérifie à l'aide d'un théorème de Weber; voir [10].

6. Nous allons donner quelques exemples numériques pour illustrer Jes quatre cas dans le Théorème 3 . $\Pi$ est toujours question de l'ensemble $[D, A, B ; E p]$.

Premier cas. Si le nombxe $D$ n'est pas un résidue quadratique modulo $p$, il n'y a aucune équation résoluble dans l'ensemble.

Deuxième aas. Pour $D=5$ et $p=11$ les équations résolubles sont

$$
x^{2}-5 y^{2}=11(x=4, y=1) \quad \text { et } \quad x^{2}-5 y^{2}=-11(x=3, y=2) \text {. }
$$

L'équation singulière est

$$
x^{2}-5 y^{2}=-1 \quad(x=2, y=1) .
$$

Powư $D=78$ et $p=11$ les équations résolubles sont

$6 x^{2}-13 y^{2}=11(x=2, y=1) \quad$ et $\quad 2 x^{2}-39 y^{2}=11(x=31, y=7)$.

L'équation singulière est

$$
3 x^{2}-26 y^{2}=1 \quad(x=3, y=1) .
$$

Pour $D=6$ et $p=29$ les équations résolubles sont

$$
2 x^{2}-3 y^{2}=29(x=4, y=1) \quad \text { et } \quad x^{2}-6 y^{2}=-29(x=5, y=3) \text {. }
$$

L'équation singulière est

$$
2 x^{2}-3 y^{2}=-1 \quad(x=y=1)
$$



sont

Troisième cas. Pour $D=55$ et $p=3$ les équations résolubles

$$
5 x^{2}-11 y^{2}=-6(x=y=1) \quad \text { et } \quad x^{2}-5 \check{5} y^{2}=-6(x=7, y=1) .
$$

L'équation singulière est

$$
5 x^{2}-11 y^{2}=1 \quad(x=3, y=2) .
$$
sont

Quatrième cas. Pour $D=15$ et $p=17$ les équations résolubles

$$
x^{2}-15 y^{2}=34:(x=7, y=1) \quad \text { et } \quad 3 x^{2}-5 y^{2}=-17(x=1, y=2) \text {. }
$$

L'équation singulière est

$$
3 x^{2}-5 y^{2}=-2 \quad(x=y=1) .
$$

Pour $D=23$ et $p=13$ les équations résolubles sont

$$
x^{2}-23 y^{2}=13(x=6, y=1) \quad \text { et } x^{2}-23 y^{2}=26 \quad(x=7, y=1) .
$$

L'équation singulière est

$$
x^{2}-23 y^{2}=2 \quad(x=5, y=1) .
$$

Finissons par un exemple où le nombre de facteurs premiers do $D$ est relativement grand. Soit $D$ égal au produit de dix nombres premiors différents impairs. Alors l'ensemble $[D, A, B ; E p]$, où $p$ est un nombre premier impair qui ne divise pas $D$, contient 2048 équations, dont an plus deux sont résolubles.

\section{§3. Le cas général d'un ensemble $[D, A, B ; E N]$}

7. Il est évident que notre méthode pour établir le Théorème 3 peut servir à démontrer un théorème analogue sur les ensembles plus généraux $[D, A, B ; E N]$, où $N$ est un nombre naturel composé. Pour: simplifier, nous nous bornons au cas dans lequel $N$ est le produit de nombres premiers impaips distincts. Les nombres $D, A, B$ ot $B$ selont définis comme plus haut. Nous supposons que $(D, N)=1$.

Cela étant, on obtiendra aisément le

THÉORËMr 4. Supposons que les nombres $D, A, B, E$ et $N$ satisfassent aux conditions indiquées ci-dessus. Alors, le nombre d'équations résolubles dans l'ensemble $[D, A, B ; E N]$ est au plus égal à $2^{s}$.

En effet, poux la démonstration on aura seulement à remplacer partout dans les paragraphes précédents le nombre $p$ par $N$. Parmi les équations appartenant à l'ensemble $[D, A, B ; E N]$ nous distinguons, comme ci-dessus, deux catégories: La première catégorie avee $B= \pm 1$; la seconde catégorie avea $E= \pm 2$.

Supposons maintenant qu'il existe deux équations résolubles appartenant à la même catégorie, savoir

$$
A x^{2}-B y^{2}=E N
$$

et

$$
A_{1} x_{1}^{2}-B_{1} y_{1}^{2}=E_{1} N \text {. }
$$

Alors on a $A B=A_{1} B_{1}=D, 1 \leqslant A<B, 1 \leqslant A_{1}<B_{1}, \quad E|=| E_{1} \mid$ et $(D, N)=1$. Les nombres $A x / y$ et $A_{1} x_{1} / y_{1}$ sont des solutions de la congruence

$$
z^{2} \equiv D(\bmod N)
$$

Nous supposons que ces nombres appartiennent à la même classe de congruences modulo $N$. Donc

$$
A_{1} x_{1} y \equiv A x y_{1}(\bmod N) \text {. }
$$

Il est bien connu que le nombre de solutions incongrues de la congruence (23) est égal à $2^{s}$; voir p.ex. [2], p. 47. Alors, Ia condition donnée par (24) entraîne qu'il y aura an plus $2^{s-1}$ possibilités pour la paịe (21) et (22), abstraction faite de la classification d'après les catégories.

Le même raisonnement s'appliquera à la paire d'équations

$$
\begin{gathered}
A x^{2}-B y^{2}=E N= \pm 2 N, \\
A_{1} x_{1}^{2}-B_{1} y_{1}^{2}=E_{1} N= \pm N,
\end{gathered}
$$

qui remplaçera la paire (19) et (20).

Par ailleurs, la démonstration se poursuivra entièrement comme dans le cas de l'ensemble $[D, A, B ; E p]$.

Cependant, il faut observer que le Théorème 4 est illusoire lorsque le nombre $s$ des facteưrs premiers de $N$ surpasse le nombre $r$, éventuellement $r+1$, des facteurs premiers de $D$.

En utilisant la théorie des idéanx dans le corps quadratique engendré par $\sqrt{D}$ on pourrait obtenir un résultat plus précis.

Remarque $x$. Il est évident que le Théorème 3 reste encore vrai si on $y$ remplace le nombre premier $p$ par une puissance de $p$. Il faut cependant ajouter la condition que $p$ ne divise aucun des produits $x y$ des inconnues.

Remarque 2. Nous avons aussi établi le résultat suivant relatif anx formes définites

$$
A x^{2}+B y^{2}=E p .
$$


Soient $A, B, D, E$ et $p$ définis comme au Théorème 3 , et soit $A<B$. Si m désigne le nombre d'équations résolubles en nombres entiers xi et y parmi les equations (25) on a ou $m=0$ ou $m=1$.

Dans le cas exceptionnel

$$
x^{2}+y^{2}=E p,
$$

où $p \equiv 1(\bmod 4)$ on a évidemment $m=2$.

Index Bibliographique

[1] A. af Ejenstam, Contributions to the thoory of the Diophantine equation $A x^{n}-B y^{n}=O$, Dissertation, Uppsala 1959.

[2] E. Landau, Vorlesungen über Zahlentheorie, Bd. 1, Loipzig 1927.

[3] W. Ljunggron, Einige Eigensehaften der Einheiten reeller quadratisehon und. rein-biquadratiseher Zahlkôrper, Vidensk. Akad. Skrifter, Matom.-naturv. klasse, nr. 12, Oslo 1936.

[4] - Solution complète de quelques équations du sixième degré à deux indéterminées, Archiv for matem. o. naturv., Bd. 48, $\mathrm{Nr}$. 7, Oslo 1946.

[5] T. Nagell, Contributions to the theory of a category of Diophantine equations of the second degree with two untenowns, Nova Aota Reg. Soc. Scient. Upsalionsis, Sor. IV, Vol. 16, No. 2, Uppsala 1955.

[6] - On a special class of Diophantine equations of the second degree, Arkiv tör matem., Bd. 3, Nr. 2, Stockholm 1954.

[7] - Solution complète de quelques équations aubiques à deux indéterminées, Jouxn. de mathém., $9^{\circ}$ sér., t. 4, Paris 1925.

[8] - Remarques sur une elasse d'équations indéterminées, Arkiv för matom., Bd. 8, Nr., Stockholm 1970.

[9] - Introduction to Number Theory, New York 1951.

[10] H. Weber, Beweis des Satzes, dass jede eigentlich primitive quadratische Form unendlich viele Primzahlen darzustellen fähig ist, Math. Annalen 20 (1882).

Regu te 14. 12. 1969

\section{Representations of real numbers by series of reciprocals of odd integers}

by
A. OpPenentm (Legon, Ghana)

Harold Davenport in memoriam

1. It is well-known that a real number $x$ between 0 and 1 can be expanded into a series of reciprocals of integers (a "sorites" of Sylvester) originally found by Lambert (see Perron [2]) as follows:

$$
x=x_{1}=\frac{1}{a_{1}}+\frac{1}{a_{2}}+\frac{1}{a_{3}}+\ldots
$$

where the positive integers $a_{i}$ are given in succession uniquely by the algorithm

$$
\text { (1.2) } \quad a_{i}=1+\left[1 / x_{i}\right], \quad x_{i+1}=x_{i}-\frac{1}{a_{i}}, \quad 0<x_{i+1}<x_{i}, \quad \ldots
$$

The process is unending: the integers $a_{i}$ satisfy the inequalities

$$
a_{i} \geqslant 2, \quad a_{i+1} \geqslant a_{i}^{2}-a_{i}+1 \quad(i \geqslant 1) .
$$

A convergent series (1.1) in which the integers $a_{i}$ satisfy (1.3) is necessarily the sylvester expansion of its sum. For rational $x$ equality must occur eventually in (1.3), i.e. for all $i>i_{0}, a_{i+1}=a_{i}^{2}-a_{i}+1$. The converse is trivially true.

I have taken the algorithm so that the process is non-ending. If we take $1 / a_{i} \leqslant x_{i}<1 /\left(a_{i}-1\right)$, the process ends for rational $x$; for irrational numbers the two processes naturally yield the same series.

Variations of (1.1) exist in which signs can be attached to the terms in accordance with prescribed rules (and appropriate changes in (1.3)).

2. Engel (anticipated by Lambert: see Perron [2]) obtained another kind of series for $x$ in $(0,1)$ :

$$
x=\frac{1}{c_{1}}+\frac{1}{c_{1} c_{2}}+\frac{1}{c_{1} c_{2} c_{3}}+\ldots
$$

